\title{
Use of the Real Time xCelligence System for Purposes of Medical Microbiology
}

\author{
ADAM F. JUNKA, ADRIANA JANCZURA, DANUTA SMUTNICKA, BEATA MĄCZYŃSKA, ANNA SECEWICZ, \\ JOANNA NOWICKA, MARZENNA BARTOSZEWICZ and GRAŻYNA GOŚCINIAK
}

Department of Microbiology, Medical University, Wrocław, Poland

Received 17 January 2012, revised 23 July 2012, accepted 24 July 2012

\begin{abstract}
Roche's xCelligence impedance-measuring instrument is one of a few commercially available systems of such type. According to the best knowledge of authors, instrument was tested so far only for eukaryotic cell research. The aim of this work was to estimate xCELLigence suitability for the microbiological tests, including (i) measurement of morphological changes in eukaryotic cells as a result of bacterial toxin activity, (ii) measurement of bacterial biofilm formation and (iii) impact of antiseptics on the biofilm structure. To test the influence of bacterial LT enterotoxin on eukaryotic cell lines, Chinese Hamster Ovary (CHO) cell line and reference strain Escherichia coli ATTC 35401 were used. To investigate Roche's instrument ability to measure biofilm formation and impact of antiseptics on its development, Staphylococcus aureus ATTC6538 reference strain was used. The data generated during the experiments indicate excellent ability of xCelligence instrument to detect cytopathic effect caused by bacterial LT endotoxin and to detect staphylococcal biofilm formation. However, interpretation of the results obtained during real-time measurement of antiseptic's bactericidal activity against staphylococcal biofilm, caused many difficulties. xCelligence instrument can be used for real-time monitoring of morphological changes in $\mathrm{CHO}$ cells treated with bacterial LT enterotoxin and for real-time measurement of staphylococcal biofilm formation in vitro. Further investigation is necessary to confirm suitability of system to analyze antiseptic's antimicrobial activity against biofilm in vitro.
\end{abstract}

Ke y w o r d s: morphological changes in $\mathrm{CHO}$ cells, staphylococcal biofilm formation in vitro, xCelligence real-time measurement

\section{Introduction}

Drug delivery systems, microrobotics and gene therapy are among the most distinctive examples of technological progress observed in medicine during recent decades. The utilization of new technologies is also observed in such medicine related areas such as medical microbiology. Nowadays, this branch of medicine uses many advanced techniques of molecular biology, like nucleic acid sequencing, cell line cultures and transgenic methods. Recently, increasing interest of microbiologists in real-time analysis techniques can be observed. Traditional methods, referred to as end point methods, allow observation or measurement of the final effect, in contrary to the real-time techniques that give insight into every phase of the experiment (Siley, 2006).

Real-time measurements of electrical impedance are the real-time techniques focusing increasing attention of microbiologists because their possible applications in the study of bacteria. Impedance is a measure of opposition to time-varying electric current in an electric element. The measure opposite to impedance is electrical conductivity describing an element's ability to conduct electric current (Charles and Sadiku, 2006). The application of impedance/conductivity sensors in microbiological assays allows to detect the presence of microorganisms or variable concentrations of medium ingredients in the reaction environment. Reduction of ion concentration (as a result of incorporation and utilization by bacteria), leads to increased impedance and decreased conductivity, and can be detected by the instrument's electrodes in real time. The number of bacterial cells or even smaller molecules like proteins or lipids can be measured after they adhere to the electrodes (Zoroub et al., 2008).

So far, impedance sensors have been used by microbiologists in basic research for a number of applications, predominantly for the evaluation of bacterial biofilm formation in vitro (Ramasamy et al., 2008). However, the studies on biofilm formation are at preliminary, experimental stage, which makes standardization and comparison of the results difficult.

There are commercially available impedance sensors dedicated for biological purposes. The newest one is the xCELLigence (Roche Diagnostics - available from: http:// www.roche-applied-science:com/sis/xcelligence/

* Corresponding author: A. Junka, Department of Microbiology, Medical University; T. Chałubińskiego 4, 50-368 Wrocław; phone: (048) 07178400 65; e-mail: junad@op.pl 


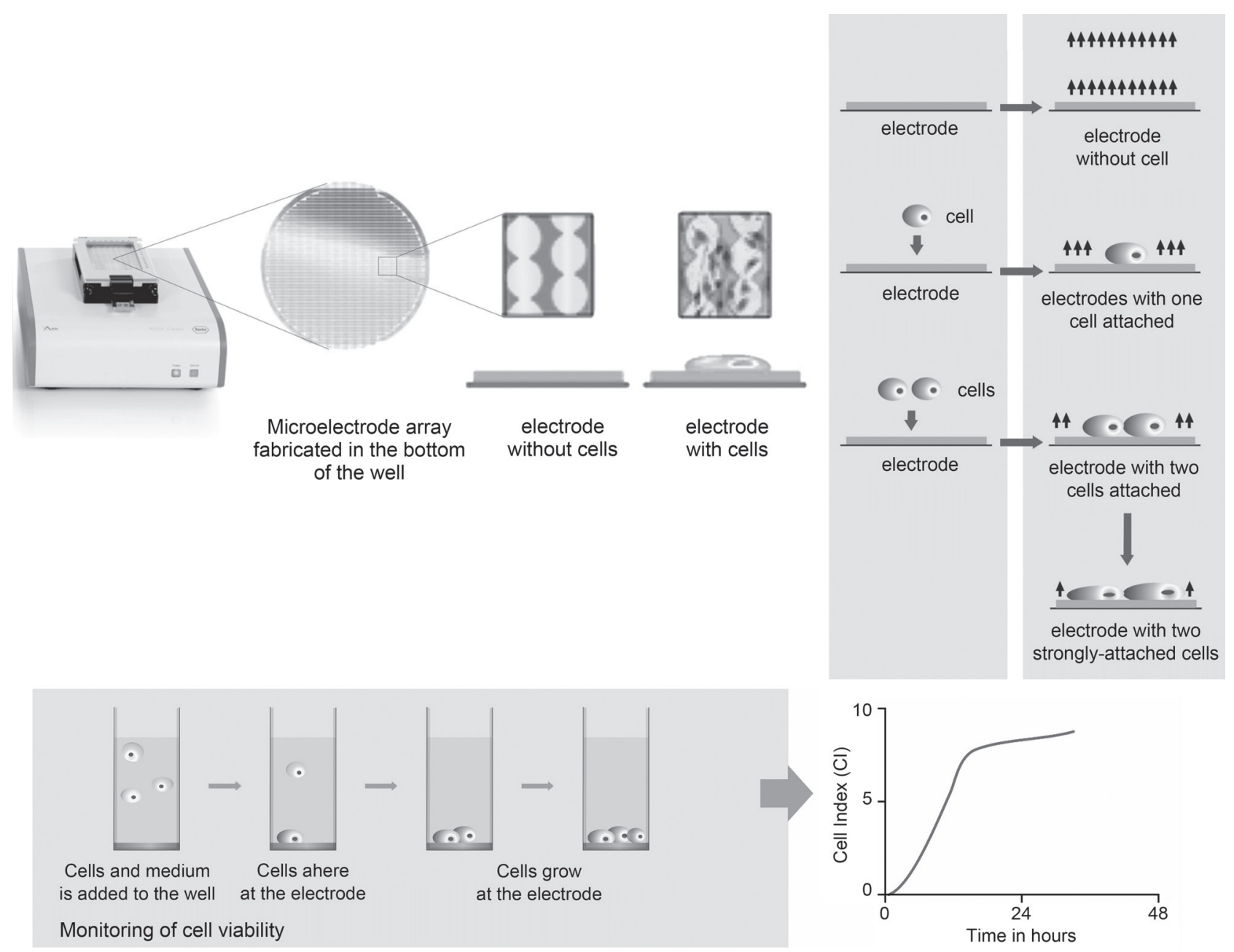

Fig. 1. Mechanism of action of Roche xCELLigence instrument

Top left side panel - xCELLigence station for real-time analysis of impedance/conductivity and ACEA Biosciences plates contain microelectrodes. Right side panel - changes in impedance caused by cells adhering to the sensors electrode. The more cells, the higher impedance and decreases conductivity. Bottom panel - impedance increase is proportional to the number of cells attached to electrodes and to the strength of adhesion. Changes in impedance are transformed into dimensionless value called Cell Index. (The figure was kindly provided by dr Rafal Kochanowski, Roche Diagnostics Poland)

ezhome.html) instrument, dedicated for the measurement of eukaryotic cell adherence and viability. Using golden microelectrodes, the xCELLigence instrument is able to detect changes in conductivity caused by adherence of eukaryotic monolayer on the bottom of the test plate (Fig. 1). Changes in impedance are transformed into dimensionless parameter termed the Cell Index (CI).

If the cells do not adhere or adhere loosely to the electrodes, the CI value equals 0 , an increase in cell adherence causes an increase in the CI value.

According to the best knowledge of the authors, the xCELLigence system has been tested and utilized only for eukaryotic cell with the major focus on the cytotoxicity and viability analysis (Blish et al., 2008, Hakki and Bozkrut, 2011, Rodrigues et al., 2011, Ge et al. 2009).

The aim of this work was to estimate xCELLigence suitability for the use in the following microbiological tests:
1. Measurement of morphological changes in eukaryotic Chinese Hamster Ovary ( $\mathrm{CHO}$ ) cell line as a result of activity of bacterial heat-labile enterotoxin (LT) produced by the Escherichia coli ATTC 35401.

2. Real-time measurement of staphylococcal (S. aureus ATCC6538 reference strain) biofilm formation.

3. Real-time measurement of bactericidal activity of cationic antiseptic Octenisept ${ }^{\natural}$ against staphylococcal biofilm (S. aureus ATCC6538).

\section{Experimental}

\section{Materials and Methods}

Measurement of morphological changes in $\mathrm{CHO}$ cell lines as a result of heat-labile LT enterotoxin activity produced by the reference E. coli strain ATTC 35401. The LT enterotoxin was produced using the method described by Speirs et al. (1977). Briefly, 
bacterial liquid culture was incubated for $16-20 \mathrm{~h}$ at $37^{\circ} \mathrm{C}$ under aerobic conditions with aeration. After incubation, the culture was centrifuged $(17000 \mathrm{~g} / 30$ $\min$ ). The supernatant was filtered through $0.45 \mu \mathrm{m}$ pore diameter membrane filters and stored at $4^{\circ} \mathrm{C}$. On the day of the experiment, the filtrate was diluted in the PBS 1:2 or 1:10.

$\mathrm{CHO}$ cells were incubated according to the manufacturer's instructions, passaged and suspended in a F12 medium. The cells were counted in a Burker chamber and diluted to the required density of $3 \times 10^{4}$ cells $/ \mathrm{ml}$. The enterotoxin's filtrates diluted 1:2 or 1:10 were added to $3 \times 10^{4} \mathrm{CHO}$ cfu and incubated for $18-24$ hours at $37^{\circ} \mathrm{C}$. The cytopathic effect causing morphological changes (elongation of cells) was observed using light microscopy (Heareus microscope, magnification x200).

Real-time assay of enterotoxin activity using Roche's xCelligence instrument. The assay was conducted in 16-well ACEA Biosciences plates containing electrodes on the bottom. Two hundred $\mu \mathrm{l}$ of $\mathrm{CHO}$ cells (density $3 \times 10^{4}$ cells $/ \mathrm{ml}$ ) were added to the appropriate wells. Next, $20 \mu \mathrm{l}$ sample of diluted toxin filtrates was introduced to the wells. The plate was incubated for $18-24$ hours at $37^{\circ} \mathrm{C}$. Changes in the Cell Index were analyzed in real time using the xCelligence instrument.

Measurement of biofilm formed by the S. aureus ATCC6538 reference strain. The ability of the S. aureus to form biofilm on different biomaterials was tested with the use of standard end-point techniques: crystal violet staining and quantitative cultures. The ability of the S. aureus strain to form biofilm on xCelligence plates was tested as follows: $200 \mu \mathrm{l}$ of medium containing $10^{5}$ bacterial cells was introduced to the appropriate wells of ACEA Biosciences plates and was incubated for $24 \mathrm{~h}$ at $37^{\circ} \mathrm{C}$. Subsequently, the bacterial suspension was removed and the wells were rinsed 3 times with sterile $0.9 \% \mathrm{NaCl}$ to remove non-adherent cells. Then, the wells were vigorously rinsed with $0.5 \%$ saponine to remove biofilm. Bacteria were serially diluted and quantitatively cultured on Columbia agar plates. Biofilm formation was estimated as $10^{4}-10^{5} \mathrm{cfu} /$ well.

Real-time measurement of biofilm formation using the xCelligence instrument. Two hundred $\mu \mathrm{l}$ of suspension containing $10^{5}$ cells of the reference $S$. aureus strain was introduced to the appropriate wells of the xCelligence instrument and incubated at $37^{\circ} \mathrm{C}$. Changes in Cell Index were measured in real time. Part "A" of the experiment: After $24 \mathrm{~h}$ of incubation, the media with non adherent cells was removed, wells were rinsed 3 times with sterile $0.9 \% \mathrm{NaCl}$ and a portion of fresh medium was added. Subsequently, the plate was incubated for additional $96 \mathrm{~h}$ to the end of the experiment ( 120 hours total) and the $\mathrm{CI}$ values were measured in real time for $120 \mathrm{~h}$. Part " $\mathrm{B}$ " of the experiment: the experiment was started as described for part $\mathrm{A}$, but the medium was not changed throughout the experiment; the plate was incubated for 126 hours.

To estimate number of cells in the biofilm, bacteria were added to the separate ACEA Biosciences plate in the same way as to the wells in experiment $A$ and $B$. After $6 \pm 1,12 \pm 1,24 \pm 1,48 \pm 1,96 \pm 1$ and $120 \pm 1$ hours from the beginning of the experiment, biofilm was washed, removed with $0.5 \%$ saponine and serial dilutions were plated on Columbia agar. The number colony forming units (CFU) was correlated with the values of $\mathrm{CI}$ measured during the real time analysis.

Real-time measurement of bactericidal activity of antiseptic against staphylococcal biofilm. S. aureus cells were incubated in wells of ACEA Biosciences plate, as described for experiments A and B. After 18 hours of incubation, when biofilm reached the plateau phase $(\mathrm{CI}=0.1)$, the medium was removed, and $200 \mu \mathrm{l}$ of Octenisept $^{\circ}$ (Schulke-Mayer) antiseptic (undiluted, $90 \%, 80 \%, 70 \%, 60 \%$ and $50 \%$ of water dilution) was introduced to the wells. CI values were measured in real time for the following 22 hours. After that time, the antiseptic was removed, the wells were rinsed, biofilm was removed with $0.5 \%$ saponine and S. aureus $\mathrm{CFU}$ count was calculated as described above.

\section{Results}

Measurement of morphological changes in $\mathrm{CHO}$ cell lines as a result of heat-labile LT enterotoxin activity produced by the reference E.coli strain ATTC 35401. The LT enterotoxin produced by Escherichia coli causes' cytopathic effect in the $\mathrm{CHO}$ lines observed as a cell elongation (Fig. 2). The traditional end-point method uses microscopy to evaluate enterotoxincaused cytopathic effect. In the traditional method, the ability of a strain to induce cytopathic effect is usually marked as "+". A lack of this ability is described as "-". If $50 \%$ of cells display morphological changes, the result is referred to as " ++ " and " +++ " corresponds to $>80 \%$ of elongated cells.

Real-time measurement of cytopathic effect using the $x$ Celligence instrument. Figure 3 presents the plot of $\mathrm{CI}$ values acquired during real-time experiment when $\mathrm{CHO}$ cells were incubated with LT toxin. The highest $\mathrm{CHO}$ cell line viability (control, red line) was observed after 9 hours from the start of the experiment (CI 0.45). After 9 hours, the viability decreased, which was reflected in decreasing $\mathrm{CI}$ values. When the $\mathrm{CHO}$ cells were treated with 1:10 enterotoxin filtrate, the highest $C I$ value $(C I=0.55)$ was observed after 12 hours from the start of the experiment. In the case of the cells treated with 1:2 enterotoxin filtrate, the CI reached the highest value $(\mathrm{CI}=0.75)$ after 11 hours from the beginning of the experiment (Fig. 3). 

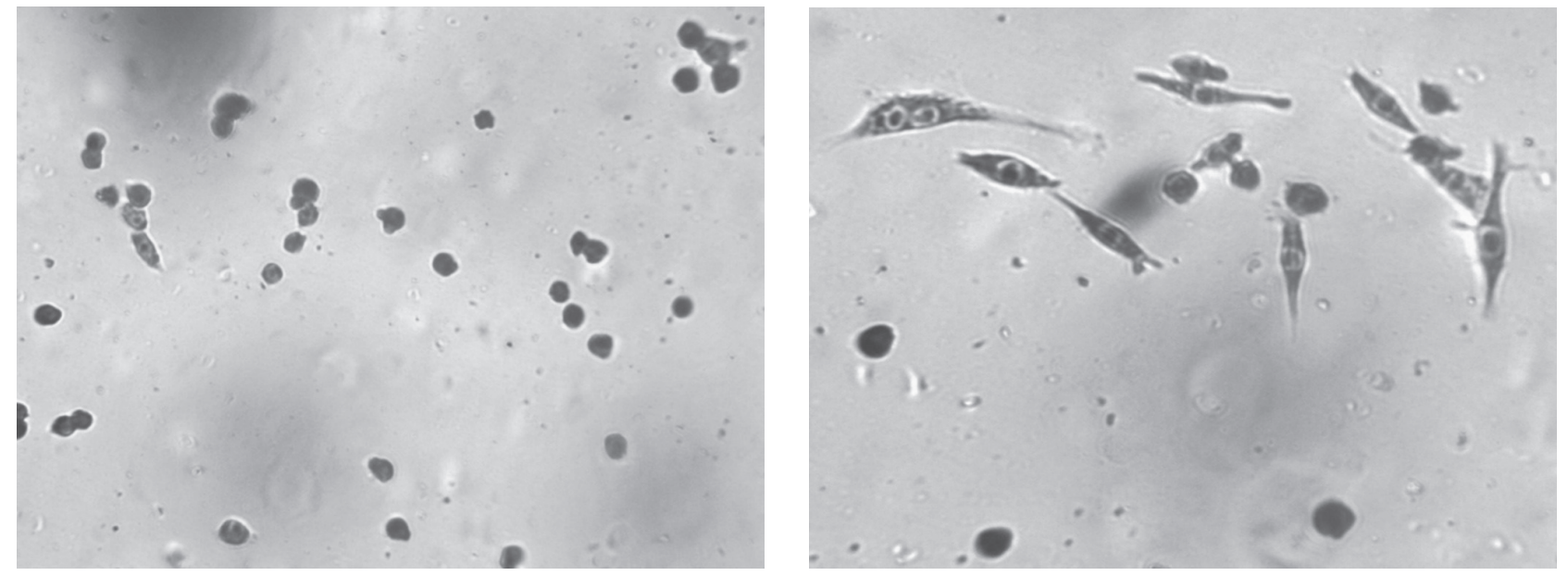

Fig. 2. Standard end-point method using light microscopy.

A - Regular CHO cells after 18 hours of incubation. B - Elongated CHO cells after 18 hours of incubation in the presence of LT enterotoxin filtrate. Heraeus microscope, magnification $\times 200$.

Real-time measurement of biofilm formed by the S. aureus ATCC6538 reference strain. Part "A" of the experiment. After 18 hours of S. aureus incubation in the well, the CI reached the highest value (0.11). A change of medium after $24 \mathrm{~h}$ of the incubation resulted in a drop in CI value to 0.075 . Between hours 24-40 of the experiment, the CI value increased (from 0.075 to 0.09 ). The CI value did not reach, as we expected, the exact value detected before medium change; however it oscillated in a closed value range (0.08-0.09) (Fig. 4).

Part "B" of the experiment. Similarly to part "A", in part "B" of the experiment, the Cell Index (CI) reached the highest value $(0.10)$ after 18 hours of incubation. Subsequently, the CI decreased, reaching 0.02 after $120 \mathrm{~h}$ of the experiment. Quantitative cultures of bacterial cells shown in Table I indicate that changes in CI values correlate with changes in the number of cells adhered to the wells of the test plate. The highest number of cells and the highest CI value were observed between the $12^{\text {th }}$ and the $24^{\text {th }}$ hour of the experiment. After 24 hours, the CI value decreased, reaching 0 after $120 \mathrm{~h}$ of the experiment. Between hours 96 to 120 , when CI reached the lowest values of 0.01 and -0.02 , the number of staphylococcal cells was also at the lower limit of detection by quantitative culturing.

Real-time measurement of bactericidal activity of antiseptic against staphylococcal biofilm using the xCelligence instrument. After 18 hours of incubation, CI reached the value of 0.1 , which correlates with the highest number of biofilm-forming cells. At that time, the medium was removed and replaced with antiseptic. During the next 20 minutes, a substantial (proportional to the concentration of antiseptic) increase in CI was

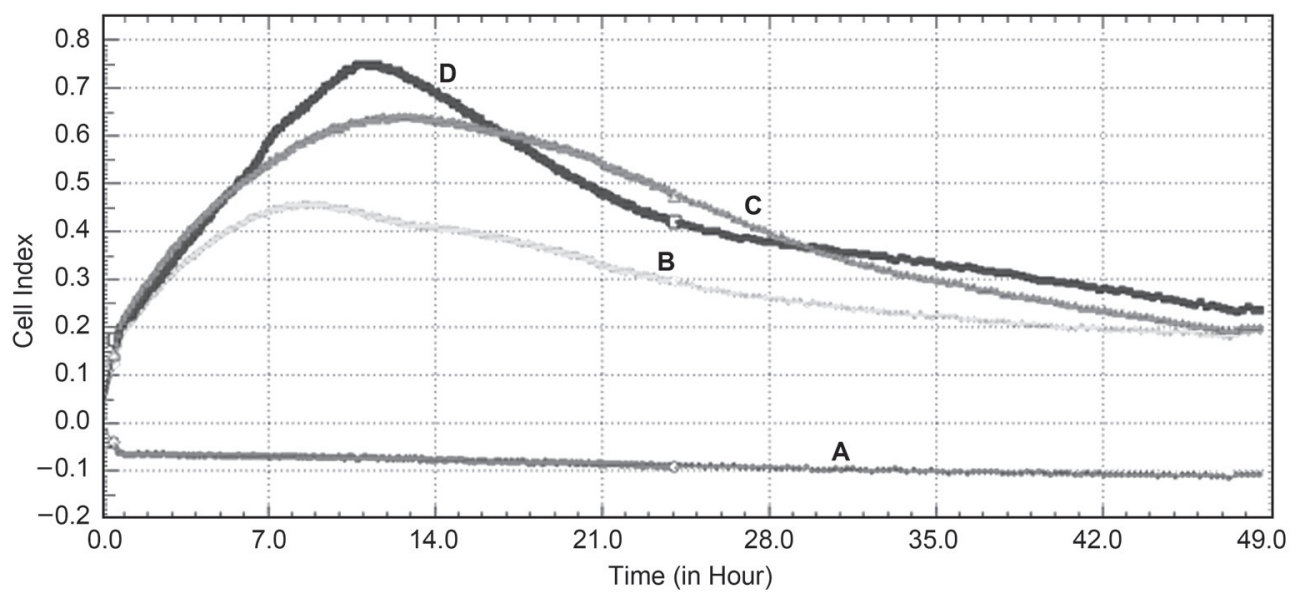

Fig. 3. Cell Index (CI) values collected in real time are a result of impedance changes caused by $\mathrm{CHO}$ cell elongation upon influence of the LT enterotoxin.

A line - negative control (F12 medium), B line - positive control, CHO cells grown in the absence of enterotoxin, $\mathrm{C}$ line - CHO cells treated with enterotoxin filtrate (diluted 1:10), D line - CHO cells treated with enterotoxin filtrate (diluted 1:2) 
Table I

Correlation between time of incubation, CI value and the number of S. aureus cells

\begin{tabular}{|l|c|c|c|c|c|c|}
\hline Time of incubation $(\mathrm{h})$ & 6 & 12 & 24 & 48 & 96 & 120 \\
\hline Cell index $(\mathrm{CI})$ & $0.04-0.06$ & $0.085-0.11$ & $0.06-0.075$ & $0.04-0.05$ & $0.00-0.01$ & $-0.02-0.0$ \\
\hline Colony forming units $(\mathrm{cfu})$ & $2.5-410^{3}$ & $3-7 \times 10^{4}$ & $9 \times 10^{3}-2 \times 10^{4}$ & $4 \times 10^{3}-7 \times 10^{3}$ & $10^{1}-10^{2}$ & $10^{1}-10^{2}$ \\
\hline
\end{tabular}

observed (Fig. 5). At the end of the experiment quantitative analysis of bacterial cell density was performed. The results of cultures were negative for the bacteria recovered from wells treated with undiluted antiseptic and $90 \%, 80 \%, 70 \%$ and $60 \%$ concentrated solutions. In the case of $50 \%$ solution, the quantitative cultures indicated the presence of less than $10^{2}$ of living bacteria. In all cases the cell index values were relatively high.

\section{Discussion}

Measurement of morphological changes in $\mathrm{CHO}$ cell lines as a result of heat-labile LT enterotoxin activity produced by the reference E.coli strain ATTC 35401. Enterotoxic Escherichia coli strains (ETEC) are able to produce heat-labile LT enterotoxin, which causes diarrhoeas in humans. Traditional methods of

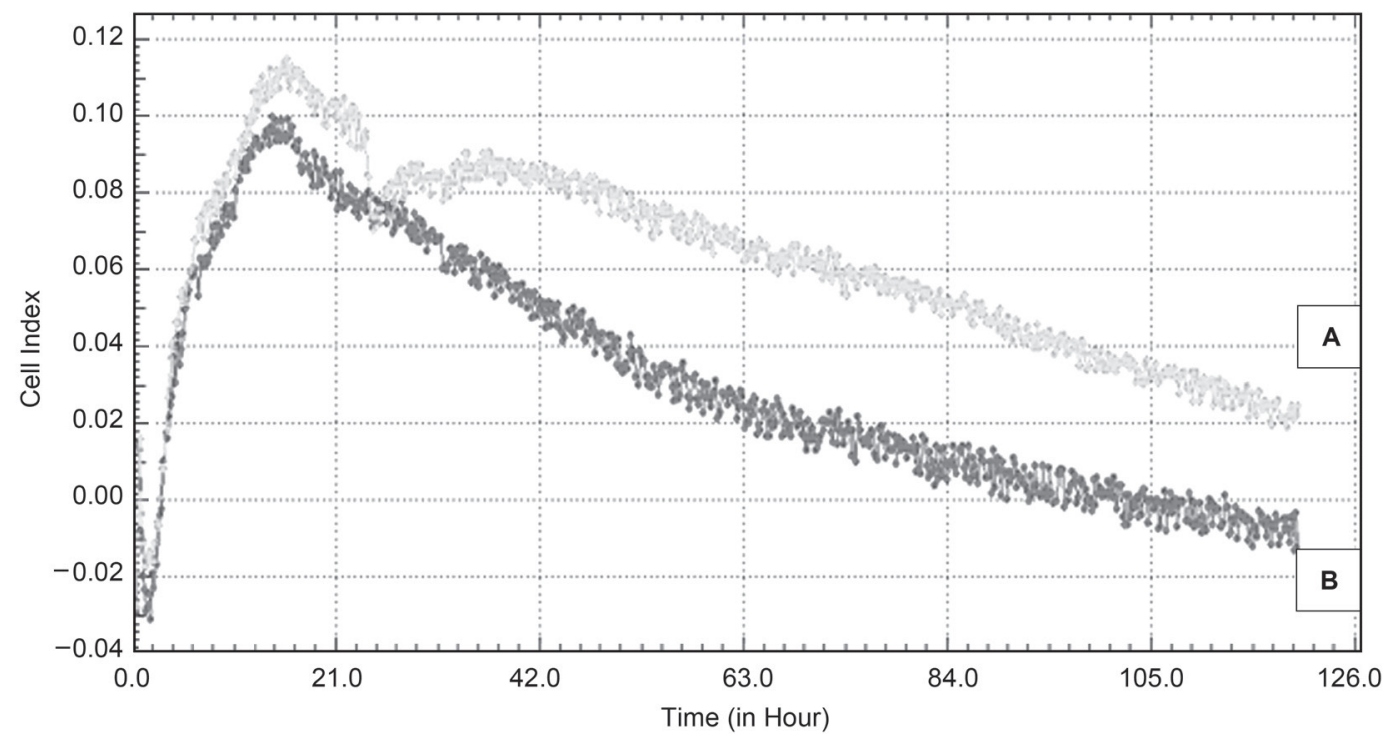

Fig. 4. Cell Index (CI) values collected in real time are a result of impedance changes caused by staphylococcal biofilm formation A. Experiment with the medium exchange, B. Experiment with the untouched biofilm.

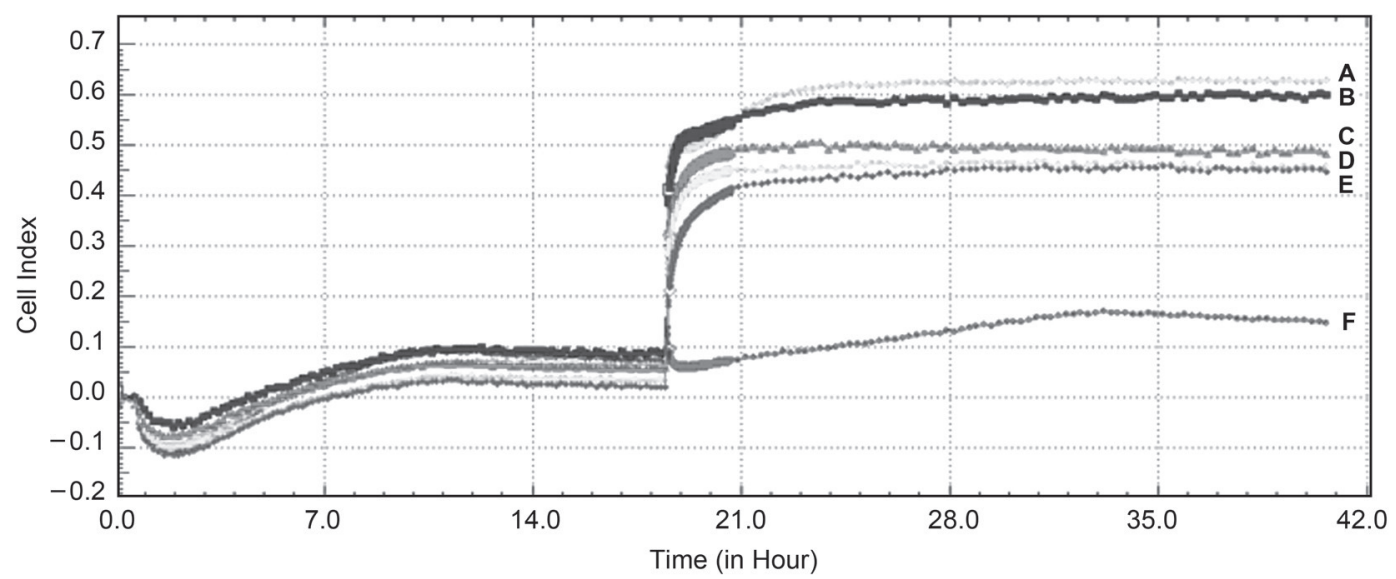

Fig. 5. Measurement of changes in CI value after treatment of S. aureus ATTC6538 biofilm with Octenisept ${ }^{ø}$ A - undiluted antiseptic, B - 90\% concentration of antiseptic, C - 80\% concentration of antiseptic, D - 70\% concentration of antiseptic, E - $60 \%$ concentration of antiseptic, $\mathrm{F}-50 \%$ concentration of antiseptic. 
LT enterotoxin detection are based on the cytopathic effect caused in $\mathrm{CHO}$ cell lines in the presence of the toxin. This cytopathic effect is visible as $\mathrm{CHO}$ cell elongation. The traditional method is an "end-point" method, which means that only the final effect can be observed or measured, leaving a whole spectrum of events undetected. In this end-point, microscopic method, the cytopathic effect was observed between 16 and 18 hours after treatment of the cells with an enterotoxin-containing filtrate. In real-time measurement, the highest CI values were observed after 11-12 hours. It means that the result obtained by the standard endpoint method does not reflect the real effect of the toxin on $\mathrm{CHO}$ cells. The use of real-time measurement allows to better estimate the influence of the toxin to influence the $\mathrm{CHO}$ cells. The cytopathic effect is gentler than the cytotoxic effects caused by other toxins or agents leading to the cell death. The presented results indicate that the xCelligence instrument was able to detect small cytopathic changes in real time and can be used for precise microbiologic diagnostics.

Real-time measurement of biofilm formed by the $S$. aureus ATCC6538 reference strain. The change of medium during biofilm incubation leads to the decrease in CI value. In studies concerning biofilm measurements in vitro, it is assumed that such action (medium change and rinsing) leads to the removal of non-adherent or loosely adherent cells (swimmer cells), which would explain the lower CI values observed during the experiment. However, it should be noted that a newly changed medium is a new source of ions in the reaction environment, which increases the value of conductivity and may decrease Cell Index. On the other hand, it is assumed that the bottom layer of biofilm is weakly permeable for a majority of molecules, hence the lower CI values may be a result of a decrease in cell number rather than of electrode-ion interactions. A precise evaluation of the observed changes needs further investigation.

The strong adherence of cells to the surface occurs during the first 4-8 hours and the rapid increase in CI value observed at this time $(-0.01-0.11)$ seems to confirm it. Initial adherence is a result of the activity of specific adhesins, after that time, adherence is mediated by exopolysaccharide - biomaterial interaction (Bjarnsholt, 2011). The cells with the exopolisaccharide attachment are at some distance from the cells of lower biofilm layers and usually are not distributed evenly in the biofilm structure. Along with the changes in the medium (decrease in nutrient concentration, increase in metabolite concentrations), the cells of the biofilms lower and mid- layer slow down their metabolic activity (anabiosis) and change their morphological properties (Dunne, 2002). An introduction of a fresh medium acti- vates "latent" bacteria and leads to the reconstruction of a biofilm structure. A decrease in $\mathrm{CI}$ values observed in part " $\mathrm{B}$ " of the experiment (hours 18 to 120) and in part " $\mathrm{A}$ " (hours 42-120) may be a result of this mechanisms; the performed quantitative cultures indicate a progressing decrease in the cell number. Because of a high number of variables, it is difficult to draw unambiguous conclusions from above data. The first aspect that should be investigated in detail is the influence of bacterial metabolic products on the electrodes' ability to conduct electric current.

Real-time measurement of bactericidal activity of antiseptic against staphylococcal biofilm using the xCelligence instrument. The analysis and interpretation of the data collected during this experiment causes some confusion. Octenidine dihydrochloride, the antiseptic's active ingredient disrupts cell continuity. In effect, ion concentration in the environment increases, which leads to decreased impedance. We expected to observe a decrease in CI value as a result of antiseptic's biocidal activity. However, after 20 minutes from the antiseptic's application, the CI increased 4-6 times. The increase in CI value was proportional to the concentration of the used antiseptic.

Sample with undiluted antiseptic was considered a background, so the observed increase in CI value is not a result of antiseptic introduction, but rather cell-electrode interaction. It has to be emphasised that impedance sensor's electrodes are placed only on the bottom of the wells. Biofilm forms not only there, but also on the walls of the well. The surface of walls is a few times larger than the surface of the bottom of the well. Probably, when antiseptic penetrates through biofilm layers, cells are disrupted and gradually are detached from the walls. Probably, cell debris sediment in the bottom of the well after treatment with antiseptic and this event is measured by the electrodes as an increase in CI value.

After real-time experiment, the presence of living cells was detected using quantitative cultures in the wells treated with $50 \%$ concentration of antiseptic only. It would be expected that the survivor cells would rebuild biofilm structure and this phenomenon would be measured by the sensor. Surprisingly, such fact was not observed. However, as it was seen in biofilm measurement assay (Result 2), the Cell Index during biofilm formation reached $0.1-0.11$. These values were lower than these reached when the antiseptic was applied. Thus, possible biofilm re-formation could be not detected by the sensor.

It is estimated that $99.9 \%$ of bacterial biomass is organized in the form of biofilm. Over $60 \%$ of all hospital infections are in fact, biofilm-related (Fux et al., 2003). The costs and burden related to the treatment 
of these infections are very high. Because every new data regarding the mechanisms of biofilm formation can be further used for biofilm eradication, research on real-time biofilm formation described in this work can be used in the future experiments.

\section{Conclusions}

\section{Real-time measurement of morphological changes} in $\mathrm{CHO}$ cell lines as a result of activity of heatlabile LT enterotoxin activity produced by the reference $E$. coli strain ATTC 35401

The experiment showed that the $\mathrm{xCelligence} \mathrm{instru-}$ ment can be used for real-time monitoring of morphological changes in $\mathrm{CHO}$ cells treated with bacterial LT enterotoxin allowing to exactly evaluate the time when the cytopathic effect occurs.

2. Real-time measurement of biofilm formed by the S. aureus ATCC6538 reference strain

The xCelligence instrument can be used in real-time measurement of biofilm formation in vitro. However, additional experiments have to be done.

3. Real-time measurement of antiseptic's bactericidal activity against staphylococcal biofilm

The large number of factors that influence the CI value during the measurement is a big caveat of the experiments of such type. We can conclude that additional experiments using substances with various mechanism of action are required.

\section{Literature}

Bjarnsholt T. 2011. Biofilm Infections. Springer Science, ISBN 978-1-4419-6083-2.

Blish R., W. Wang and M. Willingham. 2008. A human bone morphogenetic protein antagonist is down-regulated in renal cancer. Molecular Biology Cell. 19: 457-464.

Charles A. and M. Sadiku. 2006. Fundamentals of Electric Circuits (3, revised ed.). McGraw-Hill, pp. 387-389. ISBN 9780073301150. Dunne W. 2002. Seen Any Good Biofilms Lately? Clin. Microb. Rev. 15: $155-166$.

Fux C., P. Stoodley, L. Hall-Stoodley and J. Costerton. 2003. Bacterial biofilms: diagnostic and therapeutic challenge. Expert Rev. Anti-infect Ther. 1(4): 667-683.

Ge Y., Deng T. and X. Zheng. 2009. Dynamic monitoring of changes in endothelial cell-substrate adhesiveness during leukocyte adhesion by microelectrical impedance assay. Acta Biochimica et Biophysica Sinica, 41: 256-262.

Hakki S. and S. Bozkurt. 2011. Effects of different setting of diode laser on the mRNA expression of growth factors and type I collagen of human gingival fibroblasts. Lasers Med. Sci. 19: 206-221.

Ramasamy R., Z. Ren Z. and M.M. Mench. 2008. Impact of initial biofilm growth on the anode impedance of microbial fuel cells. Biotechnol. Bioeng. 1: 101-108.

Rodrigues J., C. Abramjuk and L. Vásquez. 2011. New 4-maleamic acid and 4-maleamide peptidyl chalcones as potential multitarget drugs for human prostate cancer. Pharm Res. 28: 907-919.

Siley P. 2006. Taking the impedance technique approach to real-time microbiology; Available from: www.scientistlive.com/ European-Science-News/Equipment

Speirs J.I., S. Stavric and J. Konowalchuk. 1977. Assay of Escherichia coli heat-labile enterotoxin with Vero cells. Infect. Immun. 16: 617-622.

Zoroub M., S. Elwary and A. Turner. 2008. Principals of Bacterial Detection, Springer Science, pp. 341-371. ISBN: 978-0-387-75112-2. 